\title{
Challenges and Future Prospects of Nanotechnology for Antiviral Drugs
}

\author{
Kavita Chahal $^{*}{ }^{1}$, Renu Mishra ${ }^{2}$ \\ ${ }^{1}$ Department of Botany, Government College, Chhindwara, Madhya Pradesh, India - 482011. \\ ${ }^{2}$ Department of Botany, Sri Sathya Sai College for Women, Bhopal, Madhya Pradesh, India - 462024.
}

\begin{abstract}
The emergence and re-emergence of pathogenic viruses during the last few decades are causing several pandemics. This has become a serious public health concern. The major causes could be resistance, latency, and toxicity of the virus. Also, there are other factors like improper diagnosis and immunosuppression caused by antivirals. Hence, the rapid development of antivirals following a conventional protocol of drug development is challenging. However, there is a ray of hope reflected from some alternative approaches such as gene inhibition by RNA interference (RNAi), regulation of gene expression by aptamers, ribozymes, novel drug delivery systems, in silico resources recent advances in nanomedicines. Nanotechnology-based strategies show pinkiepromise to the therapeutic management (diagnosis, treatment, and prevention) of life-threatening viral infections. The basis for the potential pharmaceutical use of nanoparticles includes their interactions with biological interfaces, their particle size, good electrical conductivity, and the Localized Surface Plasmon Resonance effect. This review discusses the strategies, challenges, drawbacks, and prospects of nanotechnology for making effective and safe antiviral drugs.
\end{abstract}

Keywords: Pathogenic viruses, nanomedicines, antiviral drugs, localized surface plasmon resonance effect.

\section{INTRODUCTION}

There have been many advances in developing newer antibiotics and antiviral agents in the last three decades. Simultaneously, pathogenic microorganisms are also developing resistance against these modern antibiotics, which is a significant concern. Our immune system must fight continuously against some viral infections. This is because many viral strains have the potential to change their antigenic structures more often. Hence, catching a cold or flu from one virus does not give you immunity against the others. Due to the high prevalence of viral infections having no specific treatment and the constant appearance of resistant strains, the development of novel antiviral agents is essential. ${ }^{1,2}$ This is accompanied by high cost, increasing toxicity, side effects, and sometimes adulteration of conventional drugs, especially in developing countries. ${ }^{3}$ Arguably, the development of safe, non-toxic, effective, and inexpensive antiviral drugs is among the top global priorities of drug development, as many viruses are not yet curable and mortality rates are high. Viruses, the most pathogenic biological structures, nanometre size, rapid mutations, and intracellular life cycle inside the host cell often make their detection difficult. In this context, nanotechnology could be a potent solution for managing life-threatening viruses by using nanoparticles and nano-based strategies for both treatment and vaccine development. ${ }^{4}$ In the present study, the most recent reviews about the strategies, challenges, drawbacks, and prospects in the field of nanotechnology and its application as antiviral drug development has been discussed.

\section{ANTIVIRAL DRUG DESIGN AND CHALLENGES}

A class of medication used for treating viral infections, with some broad-spectrum antivirals against a wide range of viruses, is called antiviral agent. ${ }^{5}$ These drugs do not destroy their target pathogen, unlike most anti-

\section{Corresponding author}

Kavita Chahal

Email : kavitachahal18@gmail.com 
biotics; instead, they inhibit their development process. ${ }^{6}$ Therapy by using antiviral drugs is challenging because viruses have rapid replication rates, rapidly changing mutations during the propagation phase, uses normal host cellular mechanisms for their functions, have poor error scanning when reading genetic code, and undergo frequent and rapid drug target mutations. Moreover, the emergence of virus resistance to antiviral drugs is of concern, and alternative treatment strategies need to be defined. ${ }^{7}$ Another challenge to antiviral drug design is the possibility of cross-reactivity of the antibodies used, increasing the risk of false positives. Sometimes the virus gets into sites like the blood-brain barrier showing less replication making its detection difficult. ${ }^{8}$

\section{STRATEGIES OF NANOTECHNOLOGY}

Nanotechnology refers to the interaction between biological systems and nanoscience with the exploitation of particles of nanometre size to prevent, diagnose, and treat various diseases. The global problem of less bioavailability of antiviral drugs can be overcome by using nanobased particles. ${ }^{9}$ Using nano-based particles is one of the most important pharmacokinetic properties of the drug delivery system that ensures the safe administration of drugs in particular doses inside the systemic circulation without undergoing any change. Regarding the management of antiviral drugs, nanotechnology-based strategies could overcome the limitations of other drug design approaches. ${ }^{10}$ To improve the pharmacokinetic and pharmacodynamic properties of a drug, nano-size particles could traverse the unreachable sites of the human body, thereby providing easy access to these sites. ${ }^{11}$ Regarding antiviral nano therapy, the most common pathway for inhibiting viral infection is the entry of the virus into the host cell. Nanoparticles can be designed to bind either to the virus or to the host cell to interrupt the interaction of the virus with the host receptor. In the RNA interference (RNAi) technology of antiviral drug design, the use of nanocarriers to cross the membrane barriers made the process much effective against viruses as these can change its surface charge. Scientific studies have revealed the use of metallic nanoparticles, silica nanoparticles, polymeric nanoparticles, carbon nanotubes, and quantum dots to be very efficient and cost-effective tools for antiviral drug delivery systems. ${ }^{12}$

\section{SENSOR-BASED NANOTECHNOLOGY}

Sensors are devices that measure a biochemical or biological event using any electronic, optical, or magnetic technology through a compact probe. Nanotechnology that is based on these sensors needs an ultra-sensitive detection system with low-cost and high-speed systems.
In this context, the following sensor systems have been developed.

- An optical or colorimetric sensor that uses gold nanoparticles functionalized with thiol-modified antisense oligonucleotides can be used against Severe Acute Respiratory Syndrome (SARS) virus. This sensor uses Point-of-care testing (POCT) to diagnose infected individuals without sending patient samples to laboratories. ${ }^{13}$ They allow detection of the analyte through simple and visible color changes.

- Electrochemical sensor that uses Graphene sheet of field-effect transistor against SARS-CoV-2 spike protein, and Gold nanoparticle functionalized carbon electrode immune-sensor against Middle East respiratory syndrome coronavirus (MERS-CoV). These sensors have high sensitivity and possibility of miniaturization. With gold nanoparticle, the functionality of biomolecules can be achieved through immobilization. ${ }^{14}$ It also has an electro-catalytic property that allows the conduction of electric response. ${ }^{15}$

- The chiral sensor uses self-assembled chiroplasmonic gold nanoparticles with quantum dots and zirconium quantum dots conjugated with magnetic nanoparticles against Infectious Bronchitis Virus (IBV). This sensor binds to the virus forming a magneto plasmonic-fluorescent nanohybrid. An external magnet can separate this hybrid and the fluorescence intensity of the separated nanohybrids was measured to determine the analyte concentration. ${ }^{16,17}$

- Reverse transcription loop-mediated isothermal amplification (RT-LAMP), with nanoparticles-based biosensors, is a method for amplifying nucleic acid. It is highly sensitive, specific, and rapid under isothermal conditions for detecting the COVID-19 virus. ${ }^{18}$

- Biomimetic magnetic nanoparticles that mimic the cell membrane of a viral host thereby help in the enrichment of virus by magnetic separation, leading to easy detection of the virus.

\section{SOME APPROVED ANTIVIRAL NANOMEDICINES}

\subsection{Organic-based nano-formulation}

\subsubsection{Polymer-based nano-formulation}

Nano-formulations that are prepared from polymers like natural hydrophilic and synthetic hydrophobic polymers are called as Polymer-based nanoformulations. For example, gelatin, albumin, and polysaccharides like alginate, dextran, and agarose. Synthetic hydrophobic polymers include the use of polylactic acid (PLA), polylactide-co-glycolic acid (PLGA), and polystyrene. ${ }^{19}$ 


\subsubsection{Nanocapsules}

A nanocapsule is a nanosized structure with a core and a shell. The drug is present in the inner core, with a polymeric shell. For example, a nanocapsule consisting of poly(iso-butylcyanoacrylate) core, entrapping azidothymidine-triphosphate (AZT-TP) polyethyleneimine, which forms the shell.

\subsubsection{Nanospheres}

Nanospheres are smaller spherical structures with the drug uniformly dispersed in the matrix. For example, Chitosan nanospheres are loaded with acyclovir.

\subsubsection{Liposomes}

It is a Lipid-based Nano formulation. Because of the properties like inert, non-toxic, easily available, cheaper, smaller size, larger surface area, and biodegradable, lipids are being used as carriers for antiviral compounds. Some examples are lecithins, triglycerides (trimyristin, tripalmetin), glyceryl palmitostearate, and fatty acid. Liposomes are small spherical vesicles produced from phospholipids that entrap an aqueous covering into which the drug is present. They can trap and deliver both hydrophilic and hydrophobic drugs. ${ }^{20}$

\subsubsection{Micelles}

Micelles are nanosized structures made of amphiphilic block copolymers; one is hydrophobic and the other hydrophilic. The hydrophobic core helps incorporate poorly water-soluble drug and the hydrophilic shell polymer acts as a barrier to protect the drug cargo and minimizes the nonspecific interactions with enzymes, proteins, and cells. For example, multifunctional poly(Llactic acid)-b-poly(ethylene glycol) (PLLA-b-PEG) copolymer modified with a sialic acid derivative was used to form polymeric micelles, and are being used to inhibit virus-mediated hemagglutination after adding Amantidine and sialic acid. ${ }^{20,21}$

\subsubsection{Dendrimers}

Dendrimers are highly branched, monodisperse threedimensional, synthetic nano-architectures, consisting of a central core, an inner shell made up of repeating units of the building blocks, and an outer shell containing numerous functionalities. ${ }^{22}$

\subsubsection{Solid lipid nanoparticles}

Solid lipid nanoparticles (SLNs) are colloidal systems composed of a solid lipid matrix-like triglycerides, partial glycerides, steroids, fatty acids, and waxes. ${ }^{23}$

\subsection{Inorganic-based nano-formulation}

\subsubsection{Gold nanoparticles}

Gold nanoparticles (AuNPs) are colloids of nanosized particles made of gold. In the presence of light, AuNP, the oscillating electromagnetic field of light triggers coherent oscillation of the free gold electrons. This process is responsible for a charge separation resulting into a dipole oscillation in the path of the electric field of light. For example, highly monodispersed gold nanoparticles, stabilized by gallic acid. inhibited Herpes Simplex Virus. ${ }^{24,25}$

\subsubsection{Silver nanoparticles}

Silver nanoparticles (AgNPs) have small size, chemical stability, catalytic activity, high conductivity, localized surface plasma resonance, and an enormous surface area, thereby facilitating rapid dissolution and, hence, possesses intrinsic antimicrobial activity. For example, carbon-coated AgNPs, poly(N-vinyl-2-pyrrolidone), and BSA-conjugated AgNPs. Used against HIV- viral surface glycoprotein. $^{26,27}$

\section{OVERCOMING DRAWBACKS OF NANOTECHNOLOGY}

Due to the smallest size of nanoparticles, the surface area increases, interparticular friction and sticking like problems become significant. Moreover, its surface charge, solubility, and the presence of functional groups may make it toxic. ${ }^{28}$ The main component of the immune system, macrophages associated with phagocytic system, may misunderstand the nanoparticles resulting in their removal from the body. ${ }^{29}$ Nanoparticles being highly reactive may produce free radicals leading to oxidative stress, inflammation, and damage to DNA, proteins, and membranes. When inside a body, it may undergo unwanted reactions that cross any cell membranes and undesirably cross the blood-brain barrier, leading to toxicity. Some limitations of the Highly Active ARV therapy (HAART) are suboptimal adherence and heavy pill burdens. Keeping the nanoparticles limitations, the corresponding safety concerns must be used in nanotechnology. Types of nanoparticles, its surface modification, dosage, toxicity, and side effects, must be reviewed for available therapeutics. $^{30}$

\section{VACCINATION WITH NANOPARTICLES}

Introducing a small amount of inactivated or attenuated antigenic material to stimulate an individual's immune system is called vaccination. ${ }^{31}$ It is the pre-emptive first line of defense against pathogens. As a result, the immune system becomes active and develops antibodies or white blood cells to combat the introduced pathogen or other allergens, resulting in adaptive immunity. It results in herd immunity and greatly improved population health in a population, with significant 
reductions in viral infection and various other diseases. In nano-vaccination, the system uses certain carriers like proteins, lipids, and polysaccharides designed to release a compound into a specific target, thereby acting like an antigen or adjuvant. ${ }^{32}$ These vaccines have the advantage of protecting antigens against premature degradation, providing sustained release, enhanced antigen stability, and providing targeted delivery of an immunogen. ${ }^{33}$

\section{FUTURE PROSPECTS OF NANOTECHNOLOGY}

The nanotechnology encompasses various advantages over the other conventional antiviral drugs. Its ultrasmall size, and large surface to volume ratio has provided many unique properties like it can incorporate drugs with surface modification, its drug delivery is well targeted and under control, it is biocompatible, it shows slow and sustained drug release and many more properties which could simplify the multidrug therapies that are currently used to treat infectious diseases. ${ }^{34}$ All of these efficiencies are necessary for a better drug delivery system against life threatening viruses. However, some modifications are required to overcome the drawbacks in the use of this technology to make it a superior antiviral drug delivery system. ${ }^{35}$ For instance, toxicity must be removed before applying the nanocarrier system. Along with it, proper dose must be determined depending upon the properties of individual nanoparticles as different nanoparticles are toxic and non-toxic under different instances and doses. Moreover, only simple, reliable, and cost-effective methods with increased bioavailability must be used to treat a greater number of heterogeneous populations. Hence, multiplexing, and multi-functionalization of the technology along with a deep understanding of the safety aspects of nanomaterials is an urgent need to achieve a concurrent antiviral drug delivery system.

\section{CONCLUSION}

Despite a relatively novel concept, nanotechnology has gained much popularity due to its various advantages over other conventional treatments and other diagnostic methods. However, to develop an effective and reliable antiviral drug delivery system, this technology's drawbacks must be overcome. Research and development must be undertaken about the safety of nanoparticles and nanotechnology against COVID-19 and any other future outbreaks. As viruses and other pathogenic microorganisms gain drug resistance, scientific research on novel antiviral technologies must gain effectiveness and dynamicity.

\section{REFERENCES}

1. Schellekens H. Antiviral method and antiviral agent (M Mori et al, JP), Biofutur 1997;165:46.

2. Bujdáková H. Photodynamic inactivation - a promising approach combating resistant microorganisms. FEMS Open Online Conference on Microbiology 2020, Belgrade, Serbia, Comenius University in Bratislava. 2020.

3. Gallo JM. Hybrid physiologically-based pharmacokinetic model for remdesivir: Application to SARS-CoV-2. Clin Transl Sci. 2021; 6 .

4. Rosa V, Ho D, Sabino-Silva R, Siqueira WL, Silikas N. Fighting viruses with materials science: Prospects for antivirus surfaces, drug delivery systems and artificial intelligence. Dent Mater. 2021;37(3):496-507.

5. Razonable RR. Antiviral drugs for viruses other than human immunodeficiency virus. Mayo Clin Proc. 2011;86(10):10091026.

6. Antiviral Agent, Definitions. 2020.

7. Ebenezer O, Jordaan MA, Damoyi N, Shapi M. Discovery of Potential Inhibitors for RNA-Dependent RNA Polymerase of Norovirus: Virtual Screening, and Molecular Dynamics. Int J Mol Sci. 2020;22(1):171.

8. Ervilla Dass. Brief review of n-acetylcysteine as antiviral agent: potential application in covid-19. J Biomed Pharm Res. 2020;9(3).

9. Wu H, Wang MD, Liang L, Xing $\mathrm{H}$, Zhang $\mathrm{CW}$, Shen $\mathrm{F}$, Huang DS, Yang T. Nanotechnology for Hepatocellular Carcinoma: From Surveillance, Diagnosis to Management. Small. 2021;17(6):e2005236.

10. Delrish E, Jabbarvand M, Ghassemi F, Amoli FA, Atyabi F, Lashay A, Soleimani M, Aghajanpour L, Dinarvand R. Efficacy of topotecan nanoparticles for intravitreal chemotherapy of retinoblastoma. Exp Eye Res. 2021;204:108423.

11. Hagaman DE, Damasco JA, Perez JVD, Rojo RD, Melancon MP. Recent Advances in Nanomedicine for the Diagnosis and Treatment of Prostate Cancer Bone Metastasis. Molecules. 2021;26(2):384.

12. Cui Y, Yan J, Wei B. Hybrid Wireframe DNA Nanostructures with Scaffolded and Scaffold-Free Modules. Angew Chem Int Ed Engl. 2021;60(17):9345-9350.

13. Baptista PV. Gold nanoprobe-based non-crosslinking hybridization for molecular diagnostics: an update. Expert Rev Mol Diagn. 2018;18(9):767-773.

14. Yang J, Li X, Du Y, Ma M, Zhang L, Zhang J, Li P. Colorimetric recognition of aromatic amino acid enantiomers by gluconic acid-capped gold nanoparticles. Amino Acids. 2021;53(2):195204.

15. Padmanabhan NT, Thomas N, Louis J, Mathew DT, Ganguly P, John $\mathrm{H}$, Pillai SC. Graphene coupled $\mathrm{TiO}_{2}$ photocatalysts for environmental applications: A review. Chemosphere. 2021;271:129506.

16. Niu X, Yang X, Li H, Liu J, Liu Z, Wang K. Application of chiral materials in electrochemical sensors. Mikrochim Acta. 2020;187(12):676

17. Moein MM. Advancements of chiral molecularly imprinted polymers in separation and sensor fields: A review of the last decade. Talanta. 2021;224:121794. 
18. Wang D. One-pot Detection of COVID-19 with Real-time Reverse-transcription Loop-mediated Isothermal Amplification (RT-LAMP) Assay and Visual RT-LAMP Assay. 2020.

19. Pradhan D, Biswasroy P, Goyal A, Ghosh G, Rath G. Recent Advancement in Nanotechnology-Based Drug Delivery System against Viral Infections. AAPS Pharm Sci Tech. 2021;22(1):47.

20. Jin Y, Zhang C, Dong XY, Zang SQ, Mak TCW. Shell engineering to achieve modification and assembly of atomicallyprecise silver clusters. Chem Soc Rev. 2021;50(4):2297-2319.

21. Bibi A, Hsu SC, Ji WF, Cho YC, Santiago KS, Yeh JM. Comparative Studies of CPEs Modified with Distinctive Metal Nanoparticle-Decorated Electroactive Polyimide for the Detection of UA. Polymers (Basel). 2021;13(2):252.

22. Zeng $\mathrm{P}, \mathrm{Xu} \mathrm{Y}$, Zeng $\mathrm{C}$, et al. Chitosan-modified poly (D, L-lactide-co-glycolide) nanospheres for plasmid DNA delivery and HBV gene-silencing. Int J Pharm. 2011;415:259-266.

23. Baram-Pinto D, Shukla S, Richman M, et al. Surface-modified protein nanospheres as potential antiviral agents. Chem Commun. 2012;48:8359-8361.

24. Connor EE, Mwamuka J, Gole A, et al. Gold nanoparticles are taken up by human cells but do not cause acute cytotoxicity. Small. 2005;1:325-327.

25. Skirtach AG, Muñoz Javier A, Kreft O, et al. Laser-induced release of encapsulated materials inside living cells. Angew Chem Int Ed Engl. 2006;45:4612-4617.

26. Rai M, Yadav A, Gade A. Silver nanoparticles as a new generation of antimicrobials. Biotechnol Adv. 2009;27:76-83.

27. Huh AJ, Kwon YJ. 'Nanoantibiotics': a new paradigm for treating infectious diseases using nanomaterials in the antibiotics resistant era. J Control Release. 2011;156:128-145.

28. Singh Om Pal, Nehru RM. Nanotechnology and Cancer Treatment. Asian J Exp Sci. 2008;22(2):45- 50
29. De Jong W, Borm P. Drug delivery and nanoparticles: Applications and hazards. Int J Nanomedicine. 2008;3(2):133-149.

30. Khiev D, Mohamed ZA, Vichare R, Paulson R, Bhatia S, Mohapatra S, Lobo GP, Valapala M, Kerur N, Passaglia CL, Mohapatra SS, Biswal MR. Emerging Nano-Formulations and Nanomedicines Applications for Ocular Drug Delivery. Nanomaterials (Basel). 2021;11(1):173.

31. Zhu W, Dong C, Wei L, Wang BZ. Promising Adjuvants and Platforms for Influenza Vaccine Development. Pharmaceutics. 2021;13(1):68.

32. Shahgolzari M, Pazhouhandeh M, Milani M, Fiering S, Khosroushahi AY. Alfalfa mosaic virus nanoparticles-based in situ vaccination induces antitumor immune responses in breast cancer model. Nanomedicine (Lond). 2021;16(2):97-107.

33. Duval KEA, Wagner RJ, Beiss V, Fiering SN, Steinmetz NF, Hoopes PJ. Cowpea Mosaic Virus Nanoparticle Enhancement of Hypofractionated Radiation in a B16 Murine Melanoma Model. Front Oncol. 2020;10:594614.

34. Zhao Y, Tong T, Wang X, Lin S, Reid EM, Chen Y. Differentiating Solutes with Precise Nanofiltration for Next Generation Environmental Separations: A Review. Environ Sci Technol. 2021;55(3):1359-1376.

35. Topcu B, Gultekinoglu M, Timur SS, Eroglu I, Ulubayram K, Eroglu H. Current approaches and future prospects of nanofibers: a special focus on antimicrobial drug delivery. J Drug Target. 2021;5:1-13.

How to cite this article: Chahal K, Mishra R. Challenges and Future Prospects of Nanotechnology for Antiviral Drugs. Int. J. Appl. Pharm. Sci. Res. (2021);6(2): 22-26. doi: https://doi.org/10.21477/ijapsr.6.2.2

Source of Support: Nil.

Conflict of Support: None declared. 